\title{
Brace efficacy: meta-analysis of studies conducted according to the SRS criteria for brace studies
}

\author{
F Zaina ${ }^{1 *}$, S Donzelli', M Lusini ${ }^{2}$, S Negrini ${ }^{3,4}$ \\ From 9th International Conference on Conservative Management of Spinal Deformities - SOSORT 2012 \\ Annual Meeting \\ Milan, Italy. 10-12 May 2012
}

\section{Background}

Bracing efficacy is questioned, since data are very variable, and comparisons are difficult, due to the lack of standard research protocols. The SRS criteria for bracing studies (SRS-C) aimed at comparing different braces, while the SOSORT Management criteria aimed at verifying the quality of brace treatment.

\section{Aim}

To compare the results of studies performed according to SRS-C, and perform a meta-analysis.

\section{Methods}

Design: systematic review and meta-analysis. Inclusion criteria: studies respecting SRS criteria for bracing studies. Protocol: an electronic search was performed in Medline to retrieve all the articles respecting the SRS-C. Data have been pooled, and subgroups made for comparisons. Odds ratios were calculated.

\section{Results}

5 studies have been included (4 retrospective, one prospective), with a total of 416 patients, Cobb Angle range 25-40', Risser 0-2, more than 10 years old, and less than 1 year post menarche at baseline. Pooling data, we had $40 \%$ of patients worsened $>36^{\circ} \mathrm{Cobb}$, including $27 \%$ with curves over $45^{\circ} ; 30 \%$ of patients were fused. Making subgroup analysis, we compared rigid braces managed according to SOSORT Criteria (SOSORT-C), with rigid braces managed without: $2 \%$ worsened (OR: 95.21 ; CI 93.7596.66), without any patients exceeding $45^{\circ}$, or fused, versus $67 \%$ worsened $\left(44 \%>45^{\circ} \mathrm{Cobb}\right)$, and $55 \%$ fused. Comparing rigid braces altogether with SpineCor, we had similar efficacy, $38 \%$ vs $42 \%$ worsened ( $24 \%$ vs $31 \%>45^{\circ}$ ), $30 \%$ vs

${ }^{1}$ ISICO (Italian Scientific Spine Institute), Milan, Italy

Full list of author information is available at the end of the article
$29 \%$ fused. We had better results for papers respecting SOSORT-C, intermediate for SpineCor, and the worst for the other rigid braces papers with significant OR.

\section{Conclusion}

Pooling data, from studies respecting the SRS-C, showed rate of efficacy that can alter favorably the natural history of AIS, $40 \%$ of worsening in high risk patients versus 60-68\% described in literature. The SOSORT-C appears fundamental to obtain good results: when they are fulfilled, progression rate is close to zero; when they are not, the efficacy is significantly lower than the one of a soft brace (SpineCor). Bracing is not only a matter of technical efficacy, but also a matter of management. Data from this meta-analysis support the use of braces to change scoliosis natural history, and reduce the rate of surgery.

\section{Author details}

${ }^{1}$ ISICO (Italian Scientific Spine Institute), Milan, Italy. ${ }^{2}$ Siena University, Siena, Italy. ${ }^{3}$ University of Brescia, Brescia, Italy. ${ }^{4}$ IRCCS Don Gnocchi, Milan, Italy.

Published: 3 June 2013

\section{References}

1. Richards BS, Bernstein RM, D'Amato CR, Thompson GH: Standardization of criteria for adolescent idiopathic scoliosis brace studies: SRS Committee on Bracing and Nonoperative Management. Spine 2005, 30(18):2068-2075, discussion 2076-2067.

2. Negrini S, Grivas TB, Kotwicki T, Rigo M, Zaina F: Guidelines on "Standards of management of idiopathic scoliosis with corrective braces in everyday clinics and in clinical research": SOSORT Consensus 2008. Scoliosis 2009, 4(1):2.

3. Lonstein JE, Carlson JM: The prediction of curve progression in untreated idiopathic scoliosis during growth. J Bone Joint Surg Am 1984, 66(7):1061-1071

doi:10.1186/1748-7161-8-S1-050

Cite this article as: Zaina et al:: Brace efficacy: meta-analysis of studies conducted according to the SRS criteria for brace studies. Scoliosis 2013 8(Suppl 1):050

(c) 2013 Zaina et al; licensee BioMed Central Ltd. This is an Open Access article distributed under the terms of the Creative Commons Attribution License (http://creativecommons.org/licenses/by/2.0), which permits unrestricted use, distribution, and reproduction in any medium, provided the original work is properly cited. 